Announcement

\title{
REVIEWERS FOR VOLUME 13
}

In addition to the editorial board, I have been fortunate to have the support of many individuals who have contributed to the review process during my first two years as editor of Law and Human Behavior. I would like to thank them for their time and thoughtful reviews.

Marilyn Bowman
Eugene Borgida
John Braithwaite
John Briere
Angela Browne
Charles Clark
Dewey Cornell
Brian Cutler
Patrick DeLeon
Tony Doob
Mary L. Durham
Donald Dutton
Joel Dvoskin
Larry Eberlein
Charles Patrick Ewing
Robert Felner
Norman J. Finkel
William Foote
Richard J. Freeman
Sol Fulero
E. Scott Geller
Paul Gendreau
Jane Goodman
Michael Gottfredson

\author{
Edith Greene \\ Kirk Heilbrun \\ Ronald F. Holler \\ Gerald Koocher \\ Felice Levine \\ R. C. L. Lindsay \\ Jean Ann Linney \\ Paul Lipsitt \\ Thomas Litwack \\ Roy Malpass \\ Dale Miller \\ Dan Perlman \\ Edward Renner \\ Caton Roberts \\ Richard Rogers \\ Vincent F. Sacco \\ J. Alexander Tanford \\ Alan Tomkins \\ Tom Tyler \\ Simon Verdun-Jones \\ Lenore Walker \\ Stephen Wasby \\ Lawrence Wrightsman \\ Dan Yarmey
}

